\title{
Interactions between co-habitating fungi elicit synthesis of Taxol from an endophytic fungus in host Taxus plants
}

\author{
Sameh S. M. Soliman ${ }^{1,2}$ and Manish N. Raizada ${ }^{1}$ * \\ ${ }^{1}$ Department of Plant Agriculture, University of Guelph, Guelph, ON, Canada \\ ${ }^{2}$ Faculty of Pharmacy, Zagazig University, Zagazig, Egypt
}

\author{
Edited by: \\ Helio K. Takahashi, Universidade \\ Federal de Sao Paulo, Brazil \\ Reviewed by: \\ Guilherme L. Sassaki, Universidade \\ Federal do Paraná, Brazil \\ Anita H. Straus, Universidade Federal \\ de Sao Paulo, Brazil \\ ${ }^{*}$ Correspondence: \\ Manish N. Raizada, Department of \\ Plant Agriculture, University of \\ Guelph, Guelph, ON, Canada N1G \\ 2W1. \\ e-mail: raizada@uoguelph.ca
}

Within a plant, there can exist an ecosystem of pathogens and endophytes, the latter described as bacterial and fungal inhabitants that thrive without causing disease to the host. Interactions between microbial inhabitants represent a novel area of study for natural products research. Here we analyzed the interactions between the fungal endophytes of Taxus (yew) trees. Fungal endophytes of Taxus have been proposed to produce the terpenoid secondary metabolite, Taxol, an anti-cancer drug. It is widely reported that plant extracts stimulate endophytic fungal Taxol production, but the underlying mechanism is not understood. Here, Taxus bark extracts stimulated fungal Taxol production 30-fold compared to a 10-fold induction with wood extracts. However, candidate plant-derived defense compounds (i.e., salicylic acid, benzoic acid) were found to act only as modest elicitors of fungal Taxol production from the endophytic fungus Paraconiothyrium SSM001, consistent with previous studies. We hypothesized the Taxus plant extracts may contain elicitors derived from other microbes inhabiting these tissues. We investigated the effects of co-culturing SSM001 with other fungi observed to inhabit Taxus bark, but not wood. Surprisingly, coculture of SSM001 with a bark fungus (Alternaria) caused a threefold increase in Taxol production. When SSM001 was pyramided with both the Alternaria endophyte along with another fungus (Phomopsis) observed to inhabit Taxus, there was an eightfold increase in fungal Taxol production from SSM001. These results suggest that resident fungi within a host plant interact with one another to stimulate Taxol biosynthesis, either directly or through their metabolites. More generally, our results suggest that endophyte secondary metabolism should be studied in the context of its native ecosystem.

Keywords: Paraconiothyrium, fungus, Taxol, Taxus, elicitor, endophyte

\section{INTRODUCTION}

Taxol is a diterpenoid anti-cancer drug harvested from Taxus (yew) trees, worth more than $\$ 2$ billion USD annually to the pharmaceutical industry (Han et al., 2007; Roberts, 2007). Taxol is found in very low concentrations in yew trees prompting the search for alternative sources (Stierle et al., 1995). Endophytes are microorganisms (fungi and bacteria) that live within a plant without causing disease symptoms. Nearly 20 years ago, it was first reported that Taxol could be synthesized by a fungal endophyte, Taxomyces andreanae, discovered living within the inner tissues of Taxus brevifolia (Stierle et al., 1993). The authenticity of fungal Taxol was confirmed using mass spectrometry, NMR, and recognition by a plant Taxol monoclonal antibody (Li et al., 1996; Moon-Jong et al., 1999). Some studies have however questioned the authenticity of fungal-derived Taxol and some controversy remains whether plant-derived compounds are required for its synthesis (Staniek et al., 2009). Several studies have shown, however, that fungal Taxol is produced independently of the plant following in vitro culturing (Li et al., 1996; Strobel et al., 1996b; Guo et al., 2006). The contradictory evidence that fungi can produce authentic Taxol and can do so autonomously may be due to variation between fungal isolates in previous studies: fungi can be polynucleate and genetically unstable. Furthermore, fungal age, storage conditions, and environment may have varied between published studies conducted in different labs. Multiple labs now claim that at least 18 different fungal genera produce Taxol (Zhou et al., 2010).

We recently cultured a novel Taxol-producing fungus living inside T. x media wood and identified it as Paraconiothyrium strain SSM001 (Soliman et al., 2011). We demonstrated that SSM001 could produce Taxol independently of plant tissues following two cycles of in vitro hyphal tip transfer and inoculation into liquid media where the fungus grew $>1000$-fold prior to peak Taxol production, all in the absence of any plant tissues or extracts (Soliman et al., 2011). The fungus was localized to the living, nutrientrich vascular tissues that radially traverse wood, known as wood medullary rays.

In general from both ecological and economic viewpoints, a microbial source of Taxol would be more useful than yew plants. However, though initially an exciting discovery, the amount of Taxol produced by the initial Taxol-producing fungus, $T$. andreanae, was found to be too low for commercial production, in the range of only micrograms per liter in liquid culture (Stierle et al., 1993; Strobel, 2003; Strobel and Daisy, 2003). Other genera of Taxol-producing fungi were subsequently found to produce similarly low levels of Taxol, including, for example: Pestalotia heterocornis, which was isolated from soil collected in a yew forest ( Noh 
et al., 1999); Pestalotiopsis microspora, a common fungus present in tropical and semitropical plants, and a widespread saprophyte on bark and decaying plant material (Metz et al., 2000); and BT2, an endophytic fungus isolated from T. chinensis var. mairei plants (Guo et al., 2006).

Several attempts have been made to enhance the production of fungal Taxol. The addition of phenylalanine, a precursor of the Taxol side-chain, increased Taxol production from plants (Veeresham et al., 2003) and fungi (Strobel et al., 1996a). Taxol production in $P$. microspora increased when phosphate was decreased and sodium benzoate was added (Li et al., 1998a). In addition, sterol biosynthesis inhibitors, such as tebuconazole and triadimefon, have been shown to increase fungal Taxol yields ( $\mathrm{Li}$ et al., 1998a). In addition to chemical elicitors, mutagenesis of Fusarium maire protoplasts by UV radiation and diethyl sulfate (DES) treatment resulted in a mutant with increased production of Taxol, with a final yield of $225.2 \mu \mathrm{g} / \mathrm{L}$ (Xu et al., 2006). Similarly, a combination of UV irradiation, ethyl methyl sulfonate (EMS), ${ }^{60} \mathrm{Co}$, and nitrosoguanidine (NTG) mutagenesis treatments of Nodulisporium sylviform, improved Taxol production from 51-126 to $314 \mu \mathrm{g} / \mathrm{L}$, resulting in strain NCEU-1 (Zhao et al., 2008; Zhou et al., 2010). Taxol output by NCEU-1 was further improved to $418 \mu \mathrm{g} / \mathrm{L}$ by UV irradiation and LiCl treatment (Zhao et al., 2008; Zhou et al., 2010). Independently, four cycles of random genome shuffling of NCEU-1 by protoplast fusion increased Taxol output from 314 to $516 \mu \mathrm{g} / \mathrm{L}$ (Zhao et al., 2008; Zhou et al., 2010).

Despite the above attempts, the largest increase ever observed in fungal Taxol production was also part of the first attempt to improve it, namely the addition of yew needle broth, which reportedly increased fungal Taxol production by 100-fold (Stierle et al., 1995). More recently, co-culture of a Taxol-producing Fusarium with Taxus suspension cells resulted in a 38-fold increase in Taxol production compared to Fusarium alone (Li et al., 2009). Despite these promising results, attempts have not been successful in discovering the underlying plant-derived elicitor(s), which may include precursors of the fungal Taxol pathway, of which only phenylalanine has been tested as noted above (Stierle et al., 1993; Strobel et al., 1996a). Clues for fungal Taxol elicitors may come from the compounds found in the nutrient-rich vascular tissue habitat of endophytic fungi, which could include various nutrient sources, other chemical factors such as plant hormones, and plant defense compounds produced in response to fungal invasion. Other candidate elicitors might be derived from organisms that share the habitat of these endophytes including other fungi.

The objective of this study was to identify novel elicitors of Taxol synthesis from the fungal endophyte Paraconiothyrium SSM001 based on compounds or organisms expected to exist in the native habitat inside Taxus plants. Our most surprising result was that interactions between co-habitating fungi can stimulate endophytic fungal Taxol production.

\section{MATERIALS AND METHODS STRAINS}

The fungal strain described in this paper, Paraconiothyrium SSM001, has been deposited at ATCC (Accession \# ATCC MYA4697).

\section{CHEMICALS}

The following reagents were from Sigma (USA), including taxane standards: Taxol (\# T7402), baccatin III (\# B8154), and cephalomannine (\# C4991); fungal nutrient media: yeast-peptonedextrose (YPD; \# Y1375) and potato-dextrose-agar (PDA; \#70139); and candidate elicitors: benzoic acid (\#242381), salicylic acid (\#S0875), methyl jasmonate (\#392707), indole acetic acid (IAA; \#15148), and resveratrol (\#R5010). Strigolactone (\#GR24) was from Dr. B. Zwanenburg (Radboud University, Nijmegen, Netherlands). All solvents used for extraction, TLC, and HPLC, were HPLC grade and obtained from Fisher Scientific.

\section{ISOLATION OF ENDOPHYTIC FUNGI}

Endophytic fungi were cultured from old branches of T. $x$ media trees grown on the Main Campus and Arboretum of the University of Guelph (Guelph, ON, Canada). Fungi were isolated from fresh plant tissues following harvesting. Taxus branches were cut into $1 \mathrm{~cm}$ long $\times 0.5 \mathrm{~cm}$ diameter sections and sterilized as follows: $2.5 \%$ sodium hypochlorite solution for $10 \mathrm{~min}$; $70 \%$ ethanol for $5 \mathrm{~min}$; washed in sterile double distilled water three times. The outer bark was stripped away and the inner tissues were further sterilized using $70 \%$ ethanol for $5 \mathrm{~min}$ followed by flaming and washing three times with sterile double distilled water. Each piece of tissue was then cut into smaller pieces $(2 \mathrm{~mm} \times 5 \mathrm{~mm})$ and cultured on PDA media in Petri plates at $25^{\circ} \mathrm{C}$ in the dark until initial fungal growth was observed. Hyphal tips were then successively transferred twice onto fresh PDA media to ensure fungal culture purity (Li et al., 1996).

\section{FUNGAL GENOTYPING}

For consistency between experiments, every fungal liquid culture was genotyped by PCR and DNA sequencing of the internal transcribed spacer regions (ITS) of 18 S rDNA (Perez-Vera et al., 2005) to confirm both strain identity and purity. The fungal mycelia were lysed by mortar and pestle with liquid nitrogen and then genomic DNA was isolated using the Plant DNeasy Kit (Qiagen, Germany) with additional adsorption of the DNA onto a silica membrane, washing, and centrifugation as previously described (Haugland et al., 2002). Fifty nanograms of the DNeasy-purified genomic DNA were added into a $20-\mu 1$ PCR reaction, with $1 \mathrm{X}$ buffer (Green GoTaq Flexi Buffer, Promega, USA, \# M8911), $1.5 \mathrm{mM} \mathrm{MgCl}_{2}$, $0.2 \mathrm{mM}$ dNTPs, 0.5 U Taq DNA polymerase (New England BioLabs, USA), and $0.2 \mu \mathrm{M}$ of each universal primer set specific for fungal ITS1 and ITS4 (Perez-Vera et al., 2005). The following conditions were used for PCR amplification: $94^{\circ} \mathrm{C}$ for $2 \mathrm{~min}$, followed by 35 cycles of: $94^{\circ} \mathrm{C}$ for $45 \mathrm{~s}, 45^{\circ} \mathrm{C}$ for $1 \mathrm{~min}$, and $72^{\circ} \mathrm{C}$ for $2 \mathrm{~min}$; with a final extension cycle of $4 \mathrm{~min}$ at $72^{\circ} \mathrm{C}$. The amplicon was purified using an Illustra GFX 96 PCR Purification kit (GE Healthcare, USA), cloned, and sequenced. Each amplicon was then aligned with the sequence of strain SSM001 using Align-BLAST software.

\section{TERMINAL RESTRICTION FRAGMENT LENGTH POLYMORPHISM ANALYSIS}

For in planta fungal DNA fingerprinting, genomic DNA from Taxus and SSM001 (positive control) were used to amplify $18 \mathrm{~S}$ rDNA using fluorescent-labeled fungal $18 \mathrm{~S}$ primers. Amplicons 
were digested with HaeIII or HheI, precipitated, mixed with $8 \mu \mathrm{l}$ GeneScan 500 TAMRA size standards (PE Applied Biosystems, UK), denatured, and loaded onto a ABI Prism310 DNA Sequencer (PE Applied Biosystems, Canada). Fragment data was collected with ABI Prism310 (v.2.0) and analyzed with Peak scanner (v.1.0).

\section{IDENTIFICATION AND QUANTIFICATION OF FUNGAL TAXOL IN LIOUID CULTURE}

Fungal tips $(\sim 10 \mathrm{mg})$ from 2 -week-old pure PDA plate cultures were cultured into $1 \mathrm{~L}$ Erlenmeyer flasks containing $500 \mathrm{ml}$ of liquid YPD media and incubated for 3 weeks in the dark at room temperature. The mycelia were filtered from the liquid media using cheesecloth. The filtered liquid media was extracted three times with $50 \mathrm{ml}$ of chloroform/methanol $(9: 1 \mathrm{v} / \mathrm{v})$. The organic layers were separated, collected, and washed using distilled water and then evaporated at $45^{\circ} \mathrm{C}$ using a Rotavapor until fully dried.

For identification of fungal Taxol by TLC, the residue from each extract was dissolved in $30 \mu \mathrm{l}$ of methanol, and $8 \mu \mathrm{l}$ applied onto silica gel plates $(10 \mathrm{~cm} \times 20 \mathrm{~cm}$, Fisher Scientific \#4861-320 and \#11028) alongside the taxane standards, Taxol, cephalomannine, and baccatin III, each at a concentration of $25 \mu \mathrm{g} / \mathrm{ml}$. TLC plates were then developed in a solvent system (chloroform/methanol, 5.0:0.5). For compound visualization, TLC plates were dipped in $0.5 \%$ vanillin/sulfuric acid reagent for $1 \mathrm{~min}$. For HPLC identification of Taxol, fungal Taxol with the same $R_{\mathrm{f}}$ value of standard Taxol was purified from several TLC plates prior to LC-MS injection. A LCQ DECA ion trap LC-MS instrument (Thermo Finnigan, USA) was used, equipped with a Finnigan SpectraSystem UV6000LP UV detector and a RESTEK Ultra Phenyl column $(250 \mathrm{~mm} \times 2.1 \mathrm{~mm}$, $5 \mu \mathrm{m})$. The binary mobile phase consisted of solvent A $(0.1 \%$ formic acid) and solvent B (acetonitrile). A gradient program was used to run the analyzed samples as follows: isocratically at $80 \% \mathrm{~A}$ for $1 \mathrm{~min}, 80-30 \%$ A for $24 \mathrm{~min}, 30-80 \%$ A for $5 \mathrm{~min}$, and isocratically at $80 \%$ A for $5 \mathrm{~min}$. The flow rate was $0.4 \mathrm{ml} / \mathrm{min}$. Taxol was measured using the UV absorbance at $233 \mathrm{~nm}$. Taxane standards were run in parallel. The electrospray (ESI) positive ion mode was used for ion detection. The system was operated as follows: shear gas and auxiliary flow rates were set at 96 and 12 (arbitrary units); voltage setting on the capillary; tube lens offset; multipole one offset; multipole two offset; lens and entrance lens were set at 32.50, $55.00,-4.40,-8.00,-14.00$, and $-58.00 \mathrm{~V}$, respectively; the capillary temperature was set at $350^{\circ} \mathrm{C}$; and the ion spray voltage was controlled at $5 \mathrm{kV}$.

Fungal Taxol was also identified and quantified using a competitive immunoassay procedure, which employed a kit (\#TA02, Hawaii Biotechnology Group, Inc., USA) in conjunction with a commercial Taxol monoclonal antibody (\#SC-69899, Santa Cruz Biotechnology Inc., USA). Manufacturer's instructions were followed. In order to buffer against interfering fungal metabolites in the fungal extract, each immunoassay read was compared to a standard curve generated each time consisting of Taxol standard added into fungal extract from non-Taxol-producing Fusarium. The specificity of the Taxol immunoassay was confirmed by testing different concentrations of diverse taxanes (baccatin III and cephalomannine) either alone or added to wells containing Taxol. HPLC of total fungal Paraconiothyrium extract was compared to diverse taxane standards to determine if they were present.
Additional details were noted in a previous paper (Soliman et al., 2011).

All Taxol concentrations were normalized to dry weight of the fungal mycelia, because we were concerned that some of the elicitors could affect the growth of the fungal mycelia, and also because we noticed flask-to-flask variation in the growth of the mycelia.

\section{PLANT-FUNGAL LIGHT MICROSCOPY}

For in vivo light microscopy, sterilized longitudinal and transverse thin wood sections were cultured on PDA media for $12 \mathrm{~h}$ followed by fixation and staining for 1 week in Trypan blue (dissolved in ethanol).

Fungal spore examination was performed by stripping off the bark and wood pieces, followed by attaching the inner side of the bark and wood to two-sided tape fixed on a microscopic slide. The tissues were pressed for a few seconds then removed, and the tape was stained with Trypan blue prior to light microscopy (Deckert et al., 2001).

\section{POST-INOCULATION TREATMENTS OF FUNGAL LIOUID CULTURES}

Fungal liquid cultures, incubated in the dark at $25^{\circ} \mathrm{C}$, with shaking at $100 \mathrm{rpm}$ (inoculation conditions as described above), were treated with either plant or fungal tissues or chemicals. For testing the effects of plant tissues, the following was added at 10 days post-inoculation: T. $x$ media wood pieces $(0.5 \mathrm{~g})$ and outer bark pieces $(0.5 \mathrm{~g})$ or their alcohol extracts $(5 \mathrm{ml})$; Pinus strobus wood pieces $(0.5 \mathrm{~g})$ and outer bark pieces $(0.5 \mathrm{~g})$ or their alcohol extracts ( $5 \mathrm{ml}$ ); and Taxus $x$ media callus suspension culture ( $30 \mathrm{ppm}$, see below). For studying the effects of non-Paraconiothyrium fungi, we isolated Alternaria and Phomopsis from T. $x$ media outer bark and needles, respectively and then added $0.2 \mathrm{~g}$ enclosed within Miracloth (Calbiochem-Novabiochem Corporation, La Jolla, CA, USA, \#475855) to a 10-day old Paraconiothyrium liquid culture. For chemical treatments, the following supplements were added at 10 days post-inoculation: benzoic acid $(0.01 \mathrm{mM})$, salicylic acid $(0.28 \mathrm{mM})$, methyl jasmonate $(200 \mathrm{mM})$, IAA $(1.01,2.02$, and $3.04 \mathrm{mM})$, strigolactone $(0.1,1.0$, and $10 \mathrm{ppm})$, and resveratrol $(0.876 \mathrm{mM})$. Each chemical was either dissolved or suspended in $1 \mathrm{ml}$ of DMSO with $4 \mathrm{ml}$ of $70 \%$ ethanol as solvent. As a control, an equal volume of solvent only was added to a parallel liquid culture. All cultures continued their incubation at $25^{\circ} \mathrm{C}$ in the dark, shaking at $100 \mathrm{rpm}$, for an additional 14 days, after which fungal Taxol was extracted as described above. The results shown represent the mean of three independent experiments (three separate flasks). Statistical comparison of means, along with all raw data, is presented in Table $\mathbf{1}$ with each statistical test noted, conducted using In Stat 3.0 (GraphPad Software, USA).

\section{CALLUS AND SUSPENSION CULTURE PROTOCOL AND TAXANE ANALYSIS}

Needles and very young stem sections of T. $x$ media were surface sterilized with $3 \%$ Clorox solution containing two drops of Tween 20 for $20 \mathrm{~min}$ with shaking. The sterilized explants were then dipped into $70 \%$ ethanol for $\sim 5 \mathrm{~min}$ and washed five times with sterile $\mathrm{ddH}_{2} \mathrm{O}$. The tissues were then cut into small explants $\left(0.5-1 \mathrm{~cm}^{2}\right)$ prior to being placed onto B5CA culture media. B5CA consists of Gamborg's B5 medium (Gamborg et al., 1968) supplemented with $0.2 \%$ casamino acids (CA), $1 \%$ sucrose, $1 \mathrm{mg} / \mathrm{L}$ 
Table 1 | Raw data of biological replicates and statistical analysis.

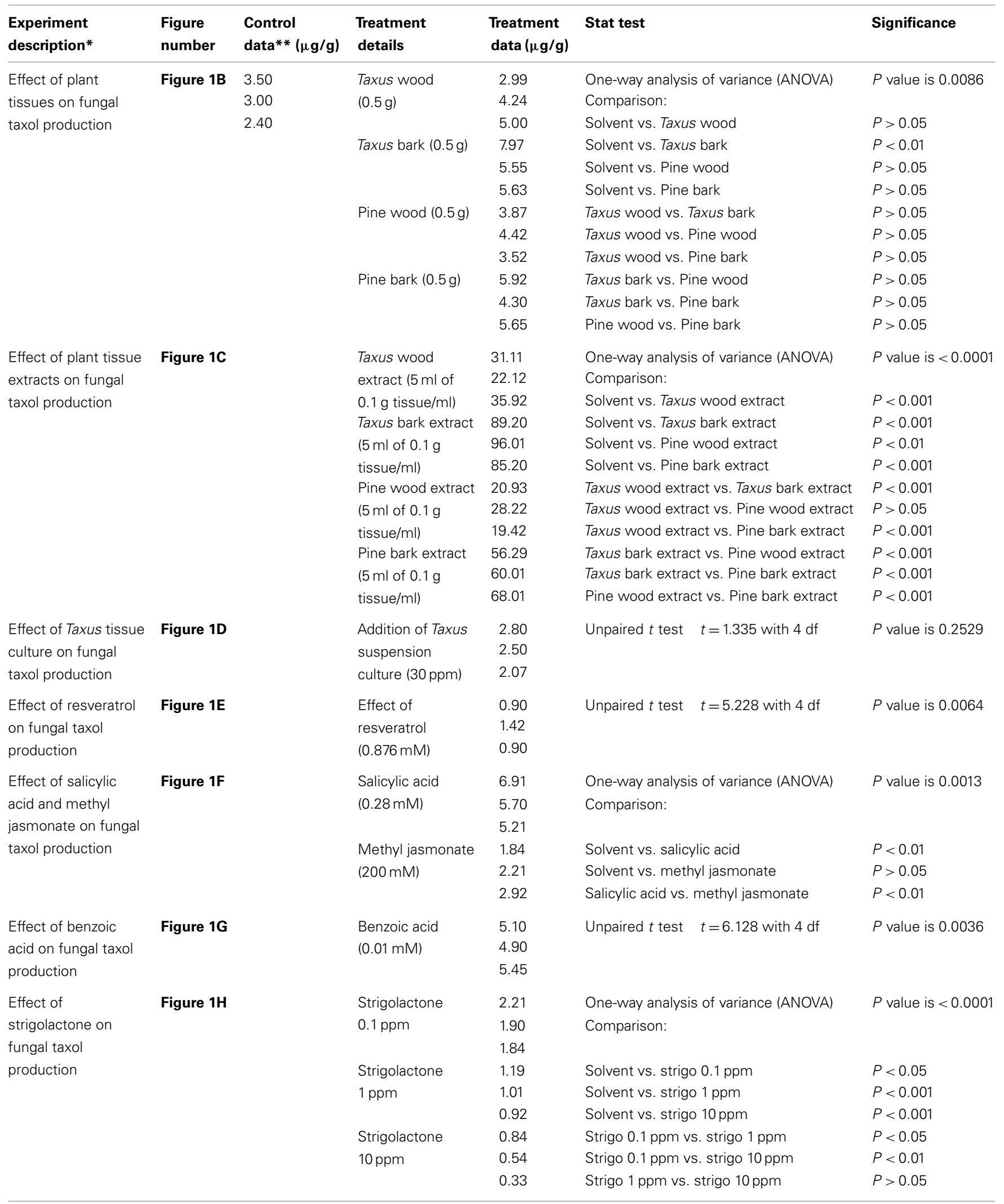


Table 1 | Continued

\begin{tabular}{|c|c|c|c|c|c|c|}
\hline $\begin{array}{l}\text { Experiment } \\
\text { description* }\end{array}$ & $\begin{array}{l}\text { Figure } \\
\text { number }\end{array}$ & $\begin{array}{l}\text { Control } \\
\text { data** }^{*}(\mu \mathrm{g} / \mathrm{g})\end{array}$ & $\begin{array}{l}\text { Treatment } \\
\text { details }\end{array}$ & $\begin{array}{l}\text { Treatment } \\
\text { data }(\mu \mathrm{g} / \mathrm{g})\end{array}$ & Stat test & Significance \\
\hline \multirow{9}{*}{$\begin{array}{l}\text { Effect of IAA on } \\
\text { fungal taxol } \\
\text { production }\end{array}$} & \multirow[t]{9}{*}{ Figure 11} & & \multirow[t]{3}{*}{ IAA $1 \mathrm{mM}$} & 0.00 & \multirow{3}{*}{$\begin{array}{l}\text { One-way analysis of variance (ANOVA) } \\
\text { Comparison: }\end{array}$} & $P$ value is $<0.0001$ \\
\hline & & & & 0.10 & & \\
\hline & & & & 0.10 & & \\
\hline & & & \multirow[t]{3}{*}{ IAA 2 mM } & 0.00 & Solvent vs. IAA $1 \mathrm{mM}$ & $P<0.001$ \\
\hline & & & & 0.05 & Solvent vs. IAA 2 mM & $P<0.001$ \\
\hline & & & & 0.09 & Solvent vs. IAA 3 mM & $P<0.001$ \\
\hline & & & \multirow[t]{3}{*}{ IAA 3 mM } & 0.00 & IAA $1 \mathrm{mM}$ vs. IAA 2 mM & $P>0.05$ \\
\hline & & & & 0.04 & IAA 1 mM vs. IAA 3 mM & $P>0.05$ \\
\hline & & & & 0.08 & IAA 2 mM vs. IAA 3 mM & $P>0.05$ \\
\hline Effect of Alternaria & \multirow[t]{3}{*}{ Figure $2 \mathrm{H}$} & & Alternaria $10.2 \mathrm{~g}$ & 9.80 & \multirow[t]{3}{*}{ Unpaired $t$ test $\quad t=4.745$ with $4 \mathrm{df}$} & \multirow[t]{3}{*}{$P$ value is 0.0090} \\
\hline on fungal taxol & & & into $500 \mathrm{ml})$ & 6.21 & & \\
\hline production & & & & 8.50 & & \\
\hline Effect of Phomopsis & \multirow[t]{3}{*}{ Figure $3 C$} & & Phomopsis $10.2 \mathrm{~g}$ & 10.20 & \multirow{3}{*}{ Unpaired $t$ test $\quad t=5.146$ with $4 \mathrm{df}$} & \multirow[t]{3}{*}{$P$ value is 0.0068} \\
\hline on fungal taxol & & & \multirow[t]{2}{*}{ into $500 \mathrm{ml}$} & 14.30 & & \\
\hline production & & & & 9.15 & & \\
\hline Effect of two fungal & Figure 4B & & Alternaria + & 20.12 & \multirow[t]{3}{*}{ Unpaired $t$ test } & \multirow[t]{3}{*}{$P$ value is 0.0004} \\
\hline cultures on fungal & & & Phomopsis $10.2 \mathrm{~g}$ & 26.30 & & \\
\hline taxol production & & & each into $500 \mathrm{ml}$ ) & 22.65 & & \\
\hline
\end{tabular}

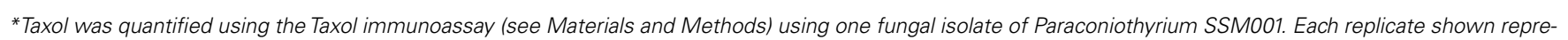
sents a pool of $500 \mathrm{ml}$ liquid fungal culture.

**All plant tissue extracts and chemical elicitors were either dissolved or suspended in ethanol:water (1:1).

2,4-D, $0.75 \%$ agar, with $\mathrm{pH}$ adjusted to 5.5 prior to autoclaving (Gibson et al., 1993). Cultures were initiated in the dark at $25^{\circ} \mathrm{C}$ and subcultured at 1-2 months intervals.

Suspension cultures were initiated from 2-month-old calli by placing $\sim 2 \mathrm{~g}$ of tissue in $1 \mathrm{~L}$ Erlenmeyer flasks containing $100 \mathrm{ml} \mathrm{B5CA}$ media at $25^{\circ} \mathrm{C}$ in dark with shaking at $100 \mathrm{rpm}$ for 2 weeks. The suspension cultures were continually grown for another 1 month. For taxane analysis, suspension cultures were filtered and the liquid media were extracted with $50 \mathrm{ml}$ chloroform: methanol (10:1) three times. The filtered, washed organic layer was then evaporated at $45^{\circ} \mathrm{C}$ under reduced pressure. The residue was then subjected to LC-MS analysis against standards Taxol and baccatin III.

\section{REAL-TIME qPCR}

RNA was isolated and PCR reaction efficiencies were determined by a series of 10 -fold dilutions using fungal $18 \mathrm{~S}$ rRNA specific primers (Fang and Bidochka, 2006), $18 \mathrm{~S}$ rRNARtF (5'-GGCATCAGTATTCAGTTGTC-3'), and 18S rRNA-RtR (5'-GTTAAGACTACGACGGTATC- $\left.3^{\prime}\right)$. Amplification conditions were as follows: $95^{\circ} \mathrm{C}$ for $10 \mathrm{~min}$, followed by 40 cycles of: denaturation, $95^{\circ} \mathrm{C}$ for $15 \mathrm{~s}$; annealing $56.6^{\circ} \mathrm{C}$ for $30 \mathrm{~s}$; extension at $72^{\circ} \mathrm{C}$ for $1 \mathrm{~min}$. The specificity of the reaction was shown by the detection of the $T_{\mathrm{m}} \mathrm{s}$ of the amplification products immediately after the last reaction cycle. Results were analyzed with the melting curve StepOne analysis software (Applied BioSystems; Soliman et al., 2011). The relative expression ratio of the fungal gene was analyzed based on real-time PCR efficiency and the crossing point differences of the samples vs. the plant 18S rRNA (Tax 18S),
Tax18SF2 (5' -TTTTCCCTTTGCAATGCC-3'), and Tax18SR2 ( $5^{\prime}$ TCGCCCTTGTAATAACCCG- $\left.3^{\prime}\right)$. The results were verified using REST (Relative Expression Software Tool) as previously described (Soliman et al., 2011).

\section{RESULTS \\ PLANT BARK AND WOOD EXTRACTS ELICIT FUNGAL TAXOL BIOSYNTHESIS}

Taxol-producing strain Paraconiothyrium SSM001 was found to be localized within T. $x$ media to the living, nutrient-rich vascular tissues that traverse dead plant wood, known as medullary rays (Figure 1A). We thus hypothesized that wood and/or bark may act as an elicitor of fungal Taxol production. We tested the effects of T. $x$ media wood and bark pieces, and their respective alcoholic extracts, on fungal Taxol production. To exclude the possibility that plant taxanes may be directly contributing to any apparent increase in fungal Taxol, pine ( $P$. strobus) was used as a control. Incubation of fungal cultures with yew bark pieces caused a modest ( $\sim$ twofold) but significant $(P<0.01)$ increase in fungal Taxol production (Figure 1B; Table 1), whereas the corresponding ethanol extracts from $500 \mathrm{mg}$ of plant tissue into $500 \mathrm{ml}$ of fungal liquid culture caused dramatic increases in fungal Taxol yield (yew wood extract, 10-fold increase; yew bark extract, 30fold; pine wood extract, eightfold; pine bark extract, 20-fold; all $P<0.01$; Figure 1C; Table 1). Because pine extracts also caused such a dramatic increase in fungal Taxol yield, this strongly suggested the increase was due to either an early precursor in the Taxol pathway or an elicitor signal. To distinguish between these two hypotheses, we applied $30 \mathrm{ppm}$ of a yew callus suspension 

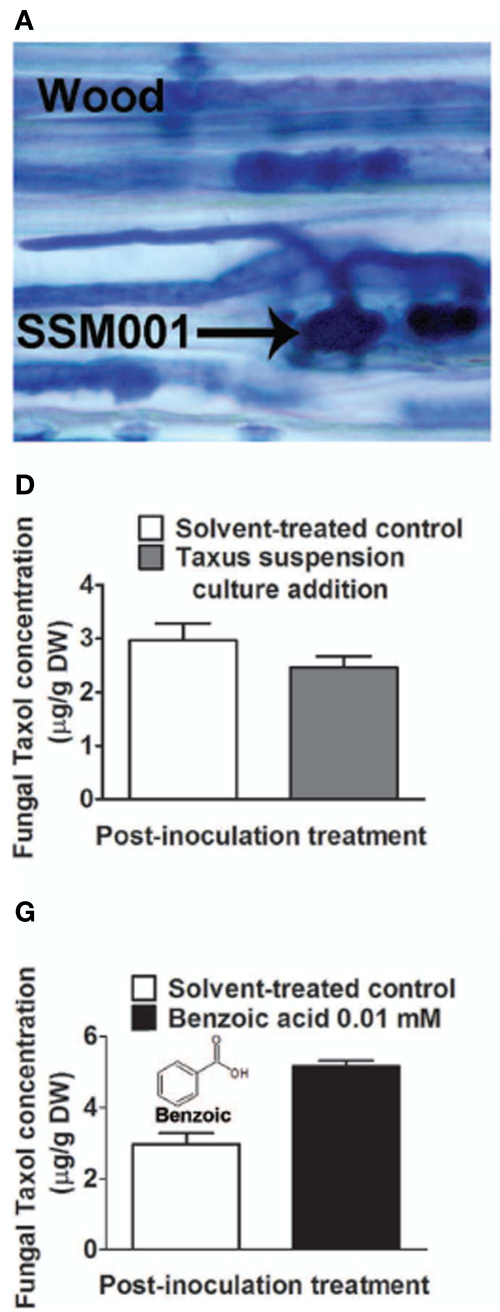

B

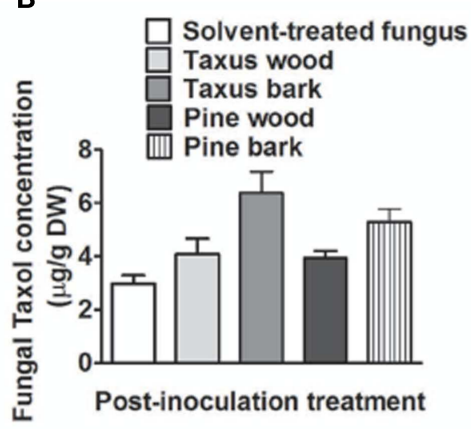

E

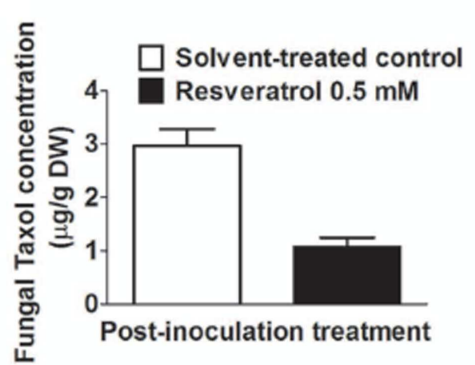

H

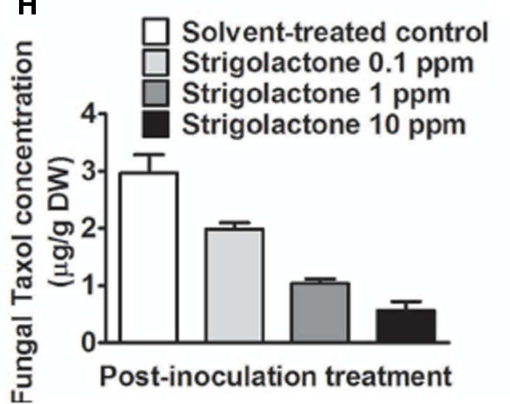

C
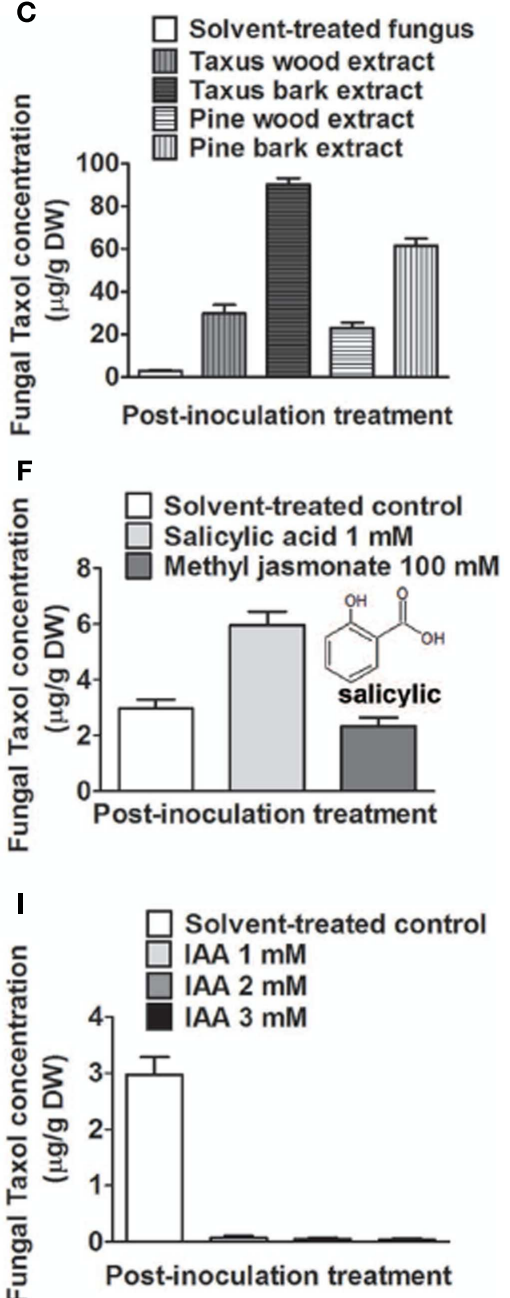

FIGURE 1 | Effect of plant extracts or candidate plant compounds, based on the habitat of Paraconiothyrium SSM001, on fungal Taxol production at $\mathbf{3}$ weeks following inoculation in fungal liquid culture. (A) Trypan

blue-stained SSM001 from 12-h-old cultured Taxus $x$ media wood sections showing SSM001 localization to the wood vascular cells (wood medullary rays). (B) Effect of added Taxus and Pinus tissues. (C) Effect of added Taxus and Pinus alcohol extracts. (D) Effect of added taxane-producing Taxus suspension culture cells to test whether Taxus contributes precursors for fungal Taxol biosynthesis. (E) Effect of the antimicrobial phytoalexin, resveratrol. (F) Effect of plant defense hormones. (G) Effect of benzoic acid, a derivative related to salicylic acid. (H) Effect of strigolactone, a plant hormone that stimulates fungal germination and penetration, and which is localized to vascular cells. (I) Effect of indole acetic acid (IAA), a plant growth hormone important in vascular tissue biogenesis. culture that showed taxane-producing activity (data not shown). The Taxus callus suspension culture did not increase fungal Taxol production (Figure 1D; $P=0.25$, Table 1), thus excluding the precursor incorporation hypothesis.

We then took a candidate approach to test for potential plantderived elicitors of fungal Taxol biosynthesis. Since Taxol has anti-fungicidal properties and Taxol biosynthesis is stimulated by pathogenic fungi (Wang et al., 2000; Strobel, 2002, 2003; Strobel and Daisy, 2003), we hypothesized that plant defense compounds synthesized in response to pathogens might stimulate fungal Taxol biosynthesis. First we tested resveratrol, an antimicrobial phytoalexin derived from the phenylpropanoid pathway, shown to be induced in pine bark in response to pathogenic fungi (Evensen et al., 2000). However, resveratrol was observed to cause a dramatic and significant $(P<0.01)$ decline rather than increase in fungal Taxol production (Figure 1E; Table 1).

Salicylic acid (Pastírová et al., 2004; Jacek et al., 2007) and methyl jasmonate (Kang et al., 2004) are plant defense hormones that stimulate protective plant secondary metabolites in response to fungal invasion (Swanty, 2000). Both hormones have been shown to stimulate plant Taxol production when added to suspension cultures of Taxus plant cells (Nims et al., 2006; Wang et al., 2007). Therefore, we hypothesized that these hormones may stimulate fungal Taxol production. Salicylic acid increased fungal Taxol production $\sim$ twofold $(P<0.01)$, whereas no significant increase was observed with methyl jasmonate (Figure 1F; Table 1). 
Previously, benzoic acid was found to increase plant Taxol production by fivefold (Arthur et al., 1994) and caused restoration of Taxol biosynthesis in fungal cultures that had lost this ability following successive re-culturing (Li et al., 1998b). Benzoic acid was suggested to be a precursor of the plant Taxol side-chain (Arthur et al., 1994), though two radiolabeling studies contradicted this interpretation (Stierle et al., 1993; Li et al., 1998b); thus the exact mechanism by which benzoic acid increases Taxol production has been unclear. Given the salicylic acid result, we noted that salicylic acid differs from benzoic acid by only a single hydroxyl group at the ortho position (Figures 1F,G). Furthermore, both compounds have been shown to have direct antifungal activity on in vitro cultured wood-inhabiting fungus Eutypa lata (Amborabé et al., 2002). We found that addition of benzoic acid to a non-attenuated culture resulted in a significant increase in fungal Taxol production, by 1.7-fold (Figure 1G; $P<0.01$, Table 1). We hypothesize that benzoic acid may be mimicking plant-derived salicylic acid.

Based on the habitat of Paraconiothyrium SSM001 in plant vascular tissues, next we tested the effects of two plant hormones likely localized to such tissues, strigolactone, and auxin (IAA). Strigolactones have classically been shown to facilitate plant-fungal communication by stimulating beneficial soil mycorrhizae fungal spore germination and hyphal penetration into host plant tissues (Akiyama and Hayashi, 2006; Yoneyama et al., 2007). More recently, strigolactone has been implicated as a hormone required for plant development, and strigolactone biosynthesis genes have been localized to plant vascular cells (Booker et al., 2005; Brewer et al., 2009). The addition of strigolactone
A

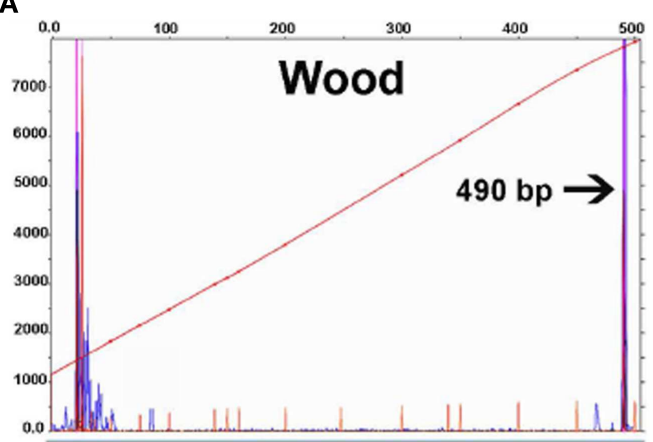

B

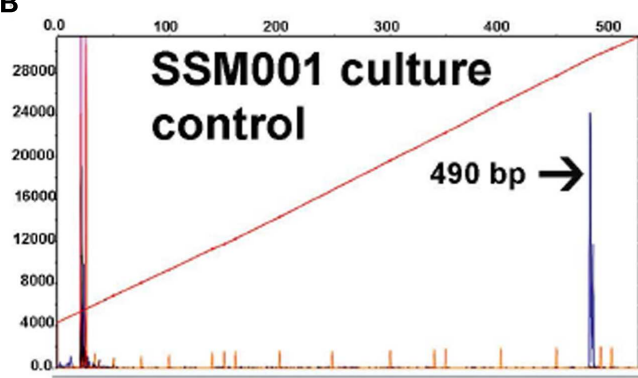

C

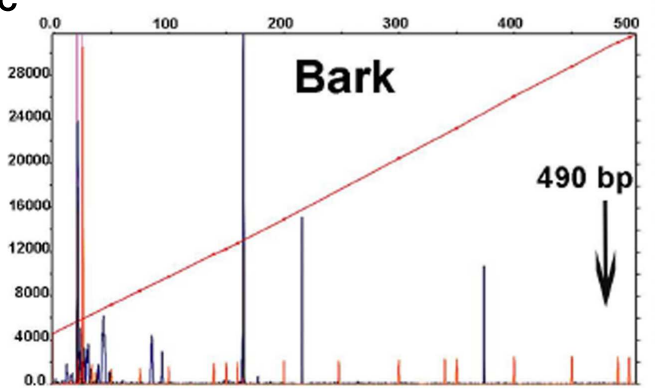

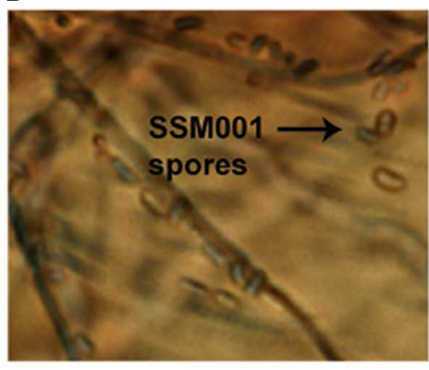

E
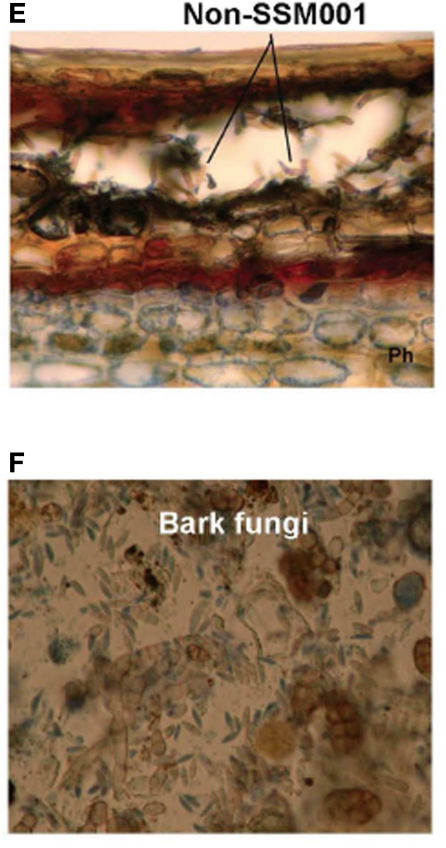

G

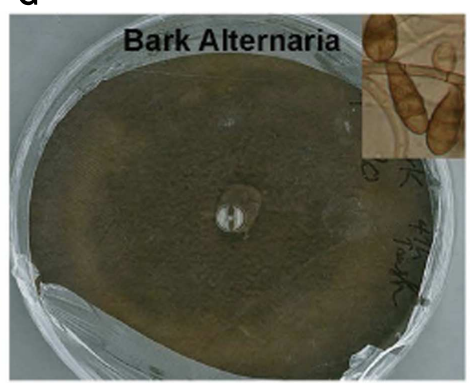

H

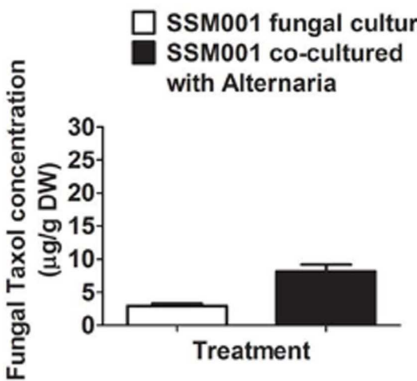

FIGURE 2 | Co-habitating fungi present in Taxus $x$ media outer bark stimulate Taxol production from Paraconiothyrium SSM001 fungi. (A) DNA fingerprinting (fungal $18 \mathrm{~S}$ tRFLP) of Taxus wood (excluding bark). The $x$-axis reflects the fragment size following Haell digestion. The dendrogram shows one peak (blue) corresponding to SSM001 along with other very minor peaks. (B) Control tRFLP of pure cultured

Paraconiothyrium SSM001 showing a peak at 490 nucleotides. (C) DNA fingerprinting (fungal 18S tRFLP) of Taxus outer bark. The dendrogram shows five novel peaks (blue) other than SSM001. (D) SSM001 spores. (E) Non-SSM001 fungal spores in the outer bark in a transverse section of a Taxus branch. (F) Close-up of spores from Taxus outer bark, stained with Trypan blue, showing the diversity of fungi. (G) Isolation and culturing of pure Alternaria fungus from the outer bark of T. x media. (H) Effect of co-culturing of pure Alternaria fungus with SSM001 in liquid culture. 
Table 2 | Sequences of fungal isolates from Taxus tissues and their taxonomic identities.

\begin{tabular}{ll}
\hline Tissue & Fungal isolate $\mathbf{1 8 S}$ rDnA \\
\hline Wood & CGAGTTTTTCNGCAAGANTTAGCATGGAATGGAATAGGACGTGCGGTCCTATTTTGTTGGTTT \\
& CTAGGACCGCCGTAATGATTAATAGGGACAGTCGGGGGCATCAGTATTCAATTGTCAGAGGTG \\
& AAATTCTTGGATTTATTGAAGACTAACTACTGCGAAAGCATTTGCCAAGGATGTTTTCATTAATC \\
& AGTGAACGAAAGTTAGGGGATCGAAGACGATCAGATACCGTCGTAGTCTTAACCATAAACTAT \\
& GCCGACTAGGGATCGGGCGGTGTTTCTATTGTGACCCGCTCGGCACCTTACGAGAAATCAAA \\
& GTGTTTGGGTTCTGGGGGGAGTATGGTCGCAAGGCTGAAACTTAAAGAAATTGACGGAAGGGCA \\
& CCACCAGGCGTGGAGCCTGCGGCTTAATTTGACTCAACACGGGGAACTCACCAGGTCCAGA
\end{tabular}

Bark TGGACCTGGNGAGTTTCCCCGTGTTGAGTCNANNNNNNNNNNNNNNTCCACGCCTGGTGGTGACC TTCCGTCAATTTCTTTAAGTTTCAGCCTTGCGACCATAATCCCCCCAGAACCCAAAAACTTTGATTTCT CGTAAGGTGCCGAGCGAGTCAGAAAAAGAACATCGCCCGATCCCTAGTCGGCATAGTTTACGGTTAA GACTACGACGGTATCTGATCGTCTTCGATCCCCTAACTTTCGTTCACTGATTAATGAAAACATCCTTGG CAAATGCTTTCGCAGTAGTTAGTCTTCAGTAAATCCAAGAATTTCACCTCTGACAACTGAATACTGATG CCCCCGACTGTTCCTGTTAATCATTGCGGCGTCTCTAGAAACCAACAAAATAGAAACGCACGTCCTAT TTCATTCCATGCTAANTCTTT

Bark AAGTAAAAGTCGTAACAAGGTCTCCGTTGGTGAACCAGCGGAGGGATCATTACCGAGTTTACAACTCC CAAACCCATGTGAACATACCTACTGTTGCTTCGGCGGGATTGCCCCGGGCGCCTCGTGTGCCCCGGA TCAGGCGCCCGCCTAGGAAACTTAATTCTTGTTTTATTTTGGAATCTTCTGAGTAGTTTTTACAAATAAAT AAAAACTTTCAACAACGGATCTCTTGGTTCTGGCATCGATGAAGAACGCAGCGAAATGCGATAAGTAAT GTGAATTGCAGAATTCAGTGAATCATCGAATCTTTGAACGCACATTGCGCCCGCCAGTATTCTGGCGGG CATGCCTGTCTGAGCGTCATTTCAACCCTCATGCCCCTAGGGCGTGGTGTTGGGGATCGGCCAAAGCC CGCGAGGGACGGCCGGCCCCTAAATCTAGTGGCGGACCCGTCGTGGCCTCCTCTGCGAAGTAGTGAT ATTCCGCATCGGAGAGCGACGAGCCCCTGCCGTTAAACCCCCAACTTTCCAAGGTTGACCTCAGATCA GGTAGGAATACCCGCTGAACTTAAGCAT

Bark AGAGGAAGTAAAAGTCGTAACAAGGTTTCCGTAGGTGAACCTGCGGAAGGATCATTACCGAGTGTAG GGTTCCTAGCGAGCCCAACCTCCCACCCGTGTTTACTGTACCTTAGTTGCTTCGGCGGGCCCGCCATT CATGGCCGCCGGGGGCTCTCAGCCCCGGGCCCGCGCCCGCCGGAGACACCACGAACTCTGTCTGA TCTAGTGAAGTCTGAGTTGATTGTATCGCAATCAGTTAAAACTTTCAACAATGGATCTCTTGGTTCCGG CATCGATGAAGAACGCAGCGAAATGCGATAACTAGTGTGAATTGCAGAATTCCGTGAATCATCGAGTC TTTGAACGCACATTGCGCCCCCTGGTATTCCGGGGGGCATGCCTGTCCGAGCGTCATTGCTGCCCAT CAAGCACGGCTTGTGTGTTGGGTCGTCGTCCCCTCTCCGGGGGGGACGGGCCCCAAAGGCAGCGG CGGCACCGCGTCCGATCCTCGAGCGTATGGGGCTTTGTCACCCGCTCTGTAGGCCCGGCCGGCGC TTGCCGAACGCAAATCAATCTTTTTCCAGGTTGACCTCGGATCAGGTAGGGATACCCGCTGAACTTAA GCATA

Bark AGTcGTAACAAGGTTTCcGTAGGTGAACCTGCGGAAGGATCATTACCGAGTTCTCGGGCTTCGGCTC GAATCTCCCACCCTTTGTGAACATACCTCTGTTGCTTTGGCGGCTCTTTGCCGCGAGGAGGCCCTC GCGGGCCCCCCCGCGCGCTTTCCGCCAGAGGACCTTCAAACTCCAGTCAGTAAACGTCGACGTCTG ATAAACAAGTTAATAAACTAAAACTTTCAACAACGGATCTCTTGGTTCTGGCATCGATGAAGAACGCA GCGAAATGCGATAAGTAATGTGAATTGCAGAATTCAGTGAATCATCGAATCTTTGAACGCACATTGCG CCCCCTGGCATTCCGGGGGGCATGCCTGTTCGAGCGTCATTACAACCCTCAAGCTCTGCTTGGTATT GGGCGCCGTCCTCTCTGCGGACGCGCCTTAAAGACCTCGGCGGTGGCTGTTCAGCCCTCAAGCGT AGTAGAATACACCTCGCTTTGGAGCGGTTGGCGTCGCCCGCCGGACGAACCTTCTGAACTTTTCTC AAGGTTGACCTCGGATCA

Bark GAAGTAAAAGTCGTAACAAGGTCTCCGTTGGTGAACCAGCGGAGGGATCATTAGAGAGTGCCCTAC TCCCAAAACCCATGTGAACTTACCTGTACGTTGCCTCGGCGGGGGAGGGGCTGGCCACCCCCCCT CCGCCAGGCGGCCCACCAAACCCTGTTTAGCCCTGAATCTCTGAGACGATAAAACAATGAGTTAAAA CTTTCAACAACGGATCTCTTGGCTCTGGCATCGATGAAGAACGCAGCGAAATGCGATACGTAGTGTG AATTGCAGAATTTAGTGAATCATCGAATCTTTGAACGCACATTGCGCCCGCCAGTATTCTGGCGGGCA TGCCTGTTCGAGCGTCATTTCAACCCCTTAAGCCCTTGTCGCTTAGTGTTGGGAGCCGACGGCGTCCT GCCGTCGCTCCTCAAATCCAGTGGCGGAGCCGGTTTCGCGCTCTGGGCGTAGTAGATTTTCTCCATCT CGCCTGCAGCCGGGGCCGGCCTCCCTGCCGTAAAACCACCACCAATGTACCCAAAGGTTGACCTCG AATCAGGTAGGAATACCCGCTGAACTTAAGCATAT

\section{Fungal species Identity value (\%)}

Paraconiothyrium 99

variabile

Alternaria $\quad 99$
alternata

Bionectria spp

100

Aspergillus

100

$\mathrm{spp}$

Diplodia spp

99

Rosellinia spp 99 


\section{Table 2 | Continued}

\begin{tabular}{|c|c|}
\hline Tissue & Fungal isolate 18S rDNA \\
\hline Bark & $\begin{array}{l}\text { TTTAGGTGANACTATAGAATACAGCGGCCGCGAGCTCGGGCCCCCACACGTGTNNNNNNNAGNN } \\
\text { ANCCTAGGCTCGAGAAGCTTGTCGACGAATTCAGATTTCTGGACCTGGTGAGTTTCCCCGTGTTGA } \\
\text { GTCAAATTAAGCCGCAGGCTCCACCCCTGGTGGTGCCCTTCCGTCAATTTCTTTAAGTTTCAGCCTT } \\
\text { GCGACCATACTCCCCCAGAACCCAAAGACTTTGATTTCTCGTAAGGTGCCGAACGGGTCAATAAG } \\
\text { TAACACCGTCCGATCCCTAGTCGGCATAGTTTATGGTTAAGACTACGACGGTATCTGATCGTCTTCGAT } \\
\text { CCCCTAACTTTCGTTCCTGATTAATGAAAACATCCTTGGCAAATGCTTTCGCAGTAGTTAGTCTTCAAT } \\
\text { AAATCCAAGAATTTCACCTCTGACAATTGAATACTGATGCCCCGACTGTCCCTATTAATCATTACGGC } \\
\text { GGTCCTAGAAACCAACAAAATAGAACCACACGTCCTATTTCATTCCATGCTAAAATCACGAATTCTGG } \\
\text { ATCCGATACGTAACGCGTCTGCAGCATGCGTGGTACCGAGCTTTCCCTATAGTGAGTCGTATTAGAGC } \\
\text { TTGGCGTAATCATGGTCATAGCTGTTTCCTGTGTGAAATTGTTATCCGCTCACAATTCCACACAACATA } \\
\text { CGAGCCGGAAGCATAAAGTGTAAAGCCTGNGGTGCCTAATGAGTGAGCTAACTCACATTAATTGCGTT } \\
\text { GCGCTCACTGCCCGCTTTCCAGTCGGGAAACCTGTCGTGCCAGCTGCATTAATGAATCGGCCAACGC } \\
\text { GCGGGGAGAGGCGGTTTGCGTATTGGGCGCTCTTCCGCTTCCTCGCTCACTGANTCGCTGCGCTCGG } \\
\text { TCGTTCGGCTGCGGCGAGCGGTATCAGCTCACTCNAAGGCGGTA }\end{array}$ \\
\hline Needles & $\begin{array}{l}\text { TAGGTGANNCTATAGAATACAGCGGCCGCGAGCTCGGGCCCCCACACGTGTGGTCTAGAGCTAGCC } \\
\text { TAGGCTCGAGAAGCTTGTCGACGAATTCAGATTTTAGCATGGAATAGAATAGGACGTGTGGTTCTATTTT } \\
\text { GTTGGTTTCTAGGACCGCCGTAATGATTAATAGGGATAGTCGGGGGCGTCAGTATTCAGCTGTCAGAGG } \\
\text { TGAAATTCTTGGATTTGCTGAAGACTAACTACTGCGAAAGCATTCGCCAAGGATGTTTTCATTAATCAGG } \\
\text { GAACGAAAGTTAGGGGATCGAAGACGATCAGATACCGTCGTAGTCTTAACCATAAACTATGCCGACTA } \\
\text { GGGATCGGACGGTGTTTCTATTATGACCCGTTCGGCACCTTACGAGAAATCAAAGTTTTTGGGTTCTGG } \\
\text { GGGGAGTATGGTCGCAAGGCTGAAACTTAAAGAAATTGACGGAAGGGCACCACAAGGCGTGGAGCCT } \\
\text { GCGGCTTAATTTGACTCAACACGGGGAAACTCACCAGGTCCAGAAATCACGAATTCTGG }\end{array}$ \\
\hline
\end{tabular}

Needles TTtAGgTGANNCTATAGAATACAGCGGCCGCGAGCTCGGGCCCCCACACGTGTGNNNTANAGCTAG CCTAGGCTCGAGAAGCTTGTCGACGAATTCAGATTTTAGCATGGAATAAAATAGGACGTCGCGGTTCTA TTTTGTTGGTTTCTAGGACCGCCGTAATGATTAATAGGGACAGTCGGGGGCATCAGTATTCAATCGTCAG AGGTGAAATTCTTGGATCGATTGAAGACTAACTACTGCGAAAGCATTTGCCAAGGATGTTTTCATTAATC AGGAACGAAAGTTAGGGGATCGAAAACGATCAGATACCGTTGTAGTCTTAACCATAAACTATGCCGACT AGGGATCGGGCGGTGTTATTTCTTGACCCGCTCGGCACCTTACACGAAAGTAAAGTTTTTGGGTTCTGG GGGGAGTATGGTCGCAAGGCTGAAACTTAAAGAAATTGACGGAAGGGCACCACAAGGGGTTAACGTT ATTGTTGCACGCAGACTCTGCCCCAGAAAGCAGCCTACGCAAGTAGGGTGTGGTGCCCTTTATATGCTA GTCGGCCGGAAGGCCGGCGATACCTTCAAATTGCGGGGATAGCCTTAGAGGCCCTGGTACCAAGCAC CACTCCGAAAGGTTGGTGTGGCGTAGCTAATCACTACGGTACGGTAATAATCCAGGTGCATTGGCCGA TCCGCAGGCAAGCCCCTCTGGGCCCCCTCGGGGGCCCCCTGTGGGAAGCTTCAGAGACTAAACGGA GGTAGGTCTGTTCGGGGAAACCCATACAGGCTTAAGATATAGTCCGAGCCAGCCCTGAAAAGGGCTG GGANGGTTGCCTTAACAAGCGCCTGANAAATGGGAGCCTGCGGCTTAATTTGACTCNACACGGGGAA ACTCACCAGGTCCAGAAATCACGAATTCTGGATCCGATACGTAAC

Needles TTAGgtgAnNCTATAGAATACAGCGgCCGCGAGCTCGgGCCCCCACACGTGTGGTCTAGAGCTAGCC TAGGCTCGAGAAGCTTGTCGACGAATTCAGATTTTAGCATGGAATAGAATAGGACGTGTGGTTCTATTTT GTTGGTTTCTAGGACCGCCGTAATGATTAATAGGGATAGTCGGGGGCGTCAGTATTCAGCTGTCAGAGG TGAAATTCTTGGATTTGCTGAAGACTAACTACTGCGAAAGCATTCGCCAAGGATGTTTTCATTAATCA GGGAACGAAAGTTAGGGGATCGAAGACGATCAGATACCGTCGTAGTCTTAACCATAAACTATGCCGACT AGGGATCGGACGGTGTTTCTATTATGACCCGTTCGGCACCTTACGAGAAATCAAAGTTTTTGGGTTCT GGGGGGAGTATGGTCGCAAGGCTGAAACTTAAAGAAATTGACGGAAGGGCACCACAAGGCGTGGA GCCTGCGGCTTAATTTGACTCAACACGGGGAAACTCACCAGGTCCAGAAATCACGAATTCTGGATCC GATACGTAACGCGTCTGCAGCATGCGTGGTACCGAGCTTTCCCTATAGTGAGTCGTATTAGAGCTTGGC GTAATCATGGTCATAGCTGTTTCCTGTGTGAAATTGTTATCCGCTCACAATTCCACACAACATACGA GCCGGAAGCATAAAGTGTAAAGCCTGGGGTGCCTAATGAGTGAGCTAACTCACATTAATTGC GTTGCGCTCACTGCCCGCTTTCCAGTCGGGAAACCTGTCGTGNCAGCTGCATTAATGAATCGGCC AACGCGCGGGGAGAGGCGGTTTGCGTATTGGGCGCTCTTCCGCTTCCTCGCTCACTGACTCGCTGCG CTCGGTCGTTCGGCTGCGGCGAGCGGNNATCAGCTCACTCNAAGGCGGTAATAC

\section{Fungal species Identity value (\%)}

Pestalotiopsis 99

spp

Paecilomyces

99

variotii

Phomopsis

99

spp

Aspergillus

99 


\section{Table 2 | Continued}

\begin{tabular}{|c|c|c|c|}
\hline Tissue & Fungal isolate 18S rDNA & Fungal species & Identity value (\%) \\
\hline \multirow{5}{*}{ Seeds } & TAGGCTCGAGAAGCTTGTCGACGAATTCAGATTTTAGCATGGAATTAAATAGGACGTGTGGTCCTATT & & \\
\hline & AGGTGAAATTCTTGGATTTATTGAAGACTAACTACTGCGAAAGCATTTGCCAAGGATGTTTTCATTAAT & & \\
\hline & CAGTGAACGAAAGTTAGGGGATCGAAGACGATCAGATACCGTCGTAGTCTTAACCGTAAACTATGCCG & & \\
\hline & ACTAGGGATCGGGCGATGTTCTTTTTCTGACTCGCTCGGCACCTTACGAGAAATCAAAGTTTTTGGGGTT & & \\
\hline & AGCCTGCGGCTTAATTTGACTCAACACGGGGAAACTCACCAGGTCCAGAA & & \\
\hline
\end{tabular}

did not increase fungal Taxol yield, but rather caused a dosedependent decline (Figure 1H; Table 1) without affecting fungal mass (data not shown). We then tested auxin as it is an important hormone required for plant vascular cell formation, including wood medullary ray biogenesis (Mattsson et al., 1999). Auxin has also been reported to stimulate conidial germination and fungal growth (Nakamura et al., 1985). Application of IAA did not increase fungal Taxol, but rather caused dramatic declines (Figure 1I, Table 1).

In conclusion, fungal Taxol increased $\sim 20-30$-fold in response to Taxus or pine bark extract. The increase in fungal Taxol was not due to plant taxane precursors, phytoalexin, or plant hormones with the exception of $\sim$ twofold increases by salicylic acid and its related compound, benzoic acid.

\section{CO-HABITATING FUNGI STIMULATE TAXOL PRODUCTION FROM PARACONIOTHYRIUM SSMO01}

Since none of the candidate plant elicitors caused a dramatic increase in fungal Taxol production, then given the fungicidal properties of Taxol (Young et al., 1992), we then hypothesized that other fungi contained within plant bark and wood may be the apparent major wood/bark elicitors of fungal Taxol production. We also hypothesized that if invasive fungi are the major elicitors of fungal Taxol production, then the reason that Taxus and pine wood had much less dramatic effects than bark on eliciting Taxol production (Table 1) may be that wood harbors a relatively small amount of non-Paraconiothyrium species. First we tested whether Taxus wood contains fungi other than the Taxolproducing Paraconiothyrium SSM001 strain. DNA isolated from Taxus wood and subjected to $18 \mathrm{~S}$ rDNA terminal restriction fragment length polymorphism (tRFLP) analysis showed only one peak at 490 bp corresponding to Paraconiothyrium SSM001, along with two very minor peaks (Figure $2 \mathrm{~A}$ ) compared to pure Paraconiothyrium tRFLP (Figure $2 \mathbf{B}$ ). In contrast, DNA isolated from Taxus bark and subjected to $18 \mathrm{~S}$ rDNA tRFLP analysis showed the presence of $>5$ fungal peaks, none of which were Paraconiothyrium SSM001 (Figure 2C) which was shown to have a peak at $\sim 490$ nucleotides (Figure 2B). Compared to pure Paraconiothyrium SSM001 spores (Figure 2D), a transverse section of a Taxus branch showed the presence of non-Paraconiothyrium spores in the outer bark (Figures 2E,F). To identify the TRFLP peaks and spore types, spores were germinated, and genomic DNA was isolated from mycelia, which was then sequenced using $18 \mathrm{~S}$ rDNA primers (Table 2). Consistent with the earlier data, only one unique endophyte was identified in Taxus wood, though it was a Paraconiothyrium species. In contrast, six unique fungi were identified in bark, including species belonging to Alternaria, Bionectria, Aspergillus, Diplodia, Rosellinia, and Pestalotiopsis (Table 2).

One of the resident bark fungi (Figure 2G) identified as an Alternaria species by spore morphology (Figure 2G, inset) and $18 S$ rDNA sequencing (Table 2) was co-cultured with Paraconiothyrium SSM001. Alone, cultures of Alternaria did not show any Taxol production using TLC or Taxol immunoassays (data not shown). However, Alternaria co-culturing caused a significant, 2.7-fold increase in Paraconiothyrium Taxol production compared to the control (Figure 2H; $P<0.01$, Table $\mathbf{1}$ ).

Since it was previously shown that aqueous yew needle extract can elicit fungal Taxol production $\leq 100$-fold, we also asked whether yew needles were a reservoir of non-Paraconiothyrium fungi. In T. $x$ media needles, we found the presence of $\sim 5-7$ distinct fungal types based on tRFLP analysis (Figure 3A) and spore morphology (Figure 3B). Culturing of the spores, followed by $18 \mathrm{~S}$ rDNA amplification and sequencing from hyphae revealed three distinct fungi belonging to the genera identified as Paecilomyces, Phomopsis, and Aspergillus (Table 2). Co-culturing Paraconiothyrium SSM001 with one needle isolate, Phomopsis, showed a significant, 3.8-fold increase in fungal Taxol (Figure 3C; $P<0.01$, Table 1). Cultures of Phomopsis alone did not show any Taxol production using TLC or Taxol immunoassays (data not shown).

Quantitative real-time PCR using specific fungal primers showed 400-fold higher concentrations of fungi in Taxus needles than in wood and 100-fold more in needles than in bark (Figure 4A). These results are consistent with at least some of the Taxol elicitor activity of Taxus needles (Stierle et al., 1995) coming from resident fungi.

To determine whether resident fungi can act in an additive manner to elicit fungal Taxol production, SSM001 was co-cultured with Alternaria isolated from Taxus bark and Phomopsis isolated from Taxus needles; co-culturing caused a 7.8-fold increase in fungal Taxol (Figure 4B; $P<0.01$, Table 1) suggesting a synergistic effect. The data suggest that, in combination with plant-derived factors, resident fungi within Taxus bark (and potentially other tissues) contribute to the strong elicitor activity of this tissue.

\section{DISCUSSION}

This study has used knowledge of the habitat (Figure 1A; Table 2) of a Taxol-producing fungus Paraconiothyrium SSM001 to systematically predict and test elicitors of fungal Taxol. We found that 
addition of extracts from the habitat of Paraconiothyrium SSM001, namely from wood and bark, caused $\sim 10-30$-fold increases in Taxol synthesis (Figures 1B,C), consistent with a previous study in which Taxus needle extracts increased fungal Taxol production by 100-fold (Stierle et al., 1995). Testing of candidate plant elicitors showed that the plant defense hormone salicylic acid, and its related compound, benzoic acid, caused twofold increases in fungal Taxol (Figure 1). A more important elicitor, however, was one of several resident non-Paraconiothyrium fungi living within elicitor outer bark (Figures 2 and 4; Table 2) suggesting that signals or other compounds released from resident pathogenic and endophytic fungi, present in extracts of bark, might contribute to the active "plant"-derived elicitor(s) of fungal Taxol, including from Taxus needles (Figure 3).

\section{CONIFER WOOD AND BARK EXTRACTS STIMULATE FUNGAL TAXOL PRODUCTION}

Since Taxus and pine tissue extracts have been shown here (Figure 1) and elsewhere (Stierle et al., 1995) to stimulate fungal Taxol production, and since some experiments with reported Taxol-producing fungi show no authentic Taxol production (Staniek et al., 2009), it has been suggested that fungi require the yew plant to synthesize Taxol. Co-culture of a Taxol-producing fungus, F. mairei, with T. chinensis, was previously shown to elicit fungal Taxol production 38-fold, but the elicitor mechanism was not tested (Li et al., 2009). We found no evidence that Taxus contributes precursors to fungal Taxol, as we observed no increase in Taxol accumulation when pure Paraconiothyrium was co-cultured with a Taxus suspension line producing taxane (Figure 1). Thus, we used knowledge of the fungal habitat in Taxus wood vascular tissues (Figure 1A) to test other plant factors. The plant hormones, strigolactone and IAA (auxin), appeared to be good candidates for a fungal Taxol elicitor since both are associated with plant vascular cells (Mattsson et al., 1999; Booker et al., 2005; Brewer et al., 2009) and both have been shown to stimulate fungal germination and growth (Nakamura et al., 1985). Surprisingly, we found that both plant hormones inhibited fungal Taxol production (Figures 1H,I). As one report had suggested that IAA can have fungicide activity (Yu et al., 2008), we tested it but observed no decrease in fungal growth (data not shown). As plants respond to fungi by producing defense compounds and hormones, we also tested resveratrol, a plant defense compound shown to be induced in conifers in response to pathogenic fungi (Evensen et al., 2000). However, we found that resveratrol strongly inhibited fungal Taxol production (Figure 1E). Resveratrol has been shown to act as a fungicide against a variety of wood decaying fungi (Seppänen et al., 2004; Välimaa et al., 2007) which might explain this result. We did, however, identify salicylic acid, an important plant defense hormone, as causing a significant, twofold increase in fungal Taxol production in the absence of any plant tissues (Figure 1F; Table 1). Interestingly, salicylic acid was previously shown to increase Taxol production in Taxus suspension cultures (Wang et al., 2004, 2007). Though limited information exists on the direct effects of salicylic acid on fungi in vitro, salicylic acid has been shown to induce mycotoxin production in F. oxysporum f. sp. niveum cultured in vitro (Wu et al., 2008). Salicylic acid has also been shown to inhibit mycelia growth, conidiation, and spores in several in vitro cultured

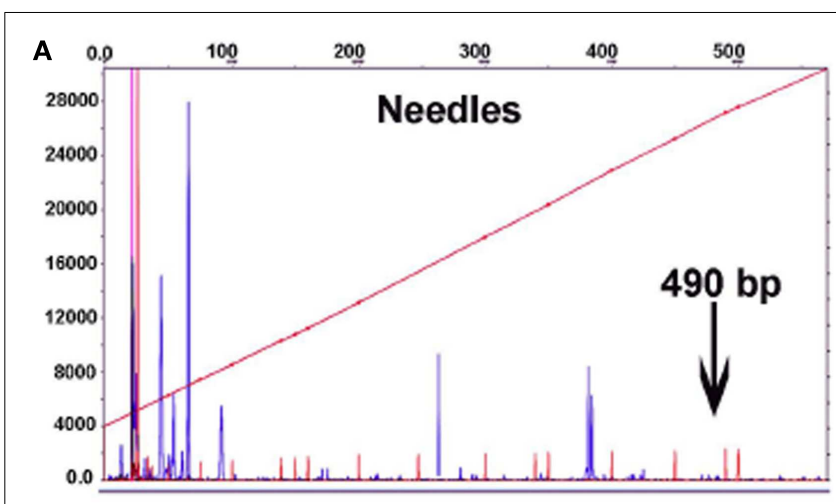

B

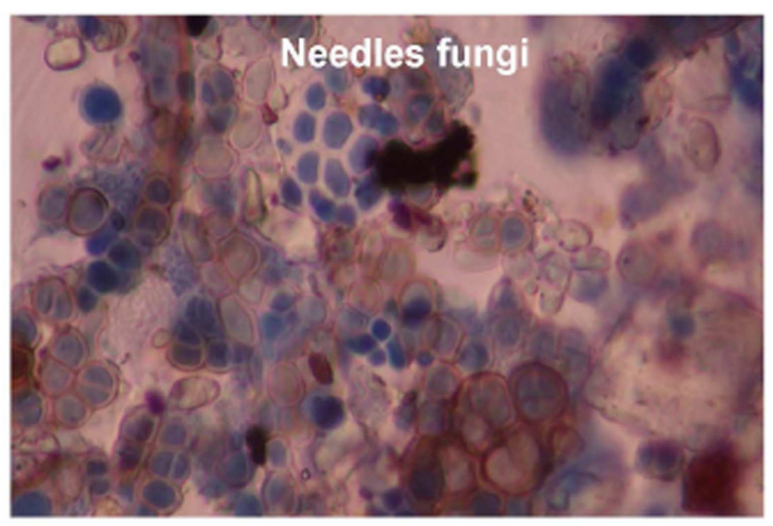

C

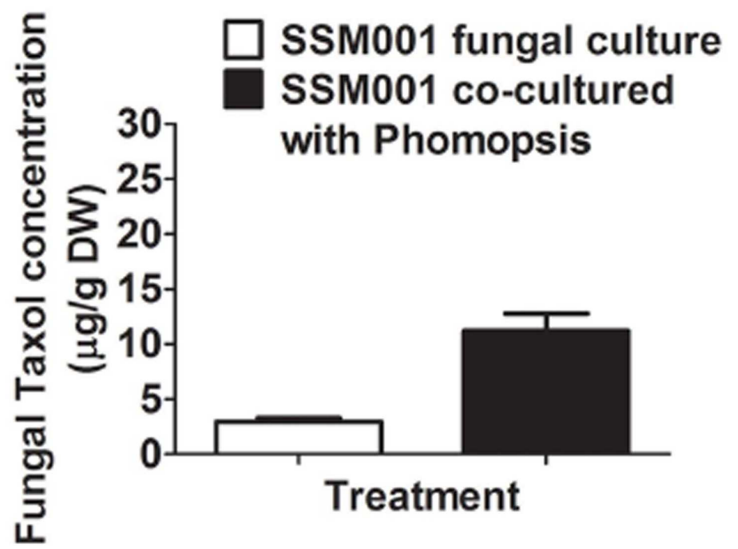

FIGURE 3 | Co-habitating fungi present in Taxus $x$ media needles stimulate Taxol production from Paraconiothyrium SSM001 fungi. (A) tRFLP analysis of DNA pooled from Taxus needles showing several fungal peaks, none of which corresponded to SSM001. (B) Detection of different fungal spores from T. $x$ media needles. (C) Effect of co-culturing of pure Phomopsis fungus isolated from Taxus needles with SSM001 in liquid culture.

fungi including Saccharomyces cerevisiae, Sphaerotheca fuliginea, Sclerotium spp, and F. oxysporum (Amborabé et al., 2002; Wu et al., 2008). Finally, we tested the effect of benzoic acid which was previously hypothesized to either restore silenced fungal Taxol 
production (Li et al., 1998b) or act as a precursor of the Taxol sidechain (Arthur et al., 1994), though radiolabeling experiments did not support the latter hypothesis (Stierle et al., 1993; Li et al., 1998b). Our results suggest a new hypothesis, that benzoic acid in fact may be mimicking the elicitor effects of the related compound, salicylic acid, which would be expected to be produced by Taxus in response to fungal colonization. Salicylic acid and benzoic acid differ by a single hydroxyl group at the ortho position of the benzene ring (Amborabé et al., 2002).

Therefore, of the 10- to 30-fold increases caused by plant extracts on fungal Taxol production (Figures 1-3; Stierle et al., 1995), we identified only one plant-derived elicitor that caused a twofold increase. Careful fractionation of Taxus extracts may be required to identify additional active plant-derived elicitors of fungal Taxol production.

\section{FUNGI RESIDENT IN TAXUS TISSUES STIMULATE TAXOL PRODUCTION FROM PARACONIOTHYRIUM}

We tested the effects of co-culturing only one of the several fungal species found in Taxus bark and discovered that it stimulated Paraconiothyrium SSM001 Taxol production 2.7-fold (Figure 2; Table 1). Subsequent co-culturing of two fungi inhabiting Taxus was shown to increase Taxol production from SSM001 by 7.8fold (Figure 4; Table 1). We thus hypothesize that the 10- to 30-fold increase in fungal Taxol caused by Taxus and pine wood and bark may in fact be due to multiple pathogenic and nonpathogenic fungi contained within these tissues acting together as elicitors. As to why bark and needles were observed to act as greater elicitors than wood of fungal Taxol production, one possibility is that wood has a low titer of fungi. Consistent with this hypothesis, our tRFLP analysis and direct $18 \mathrm{~S}$ rDNA sequencing of cultured spores demonstrated that Taxus bark and needles have several non-Paraconiothyrium fungi compared to wood (Figures 2 and 3; Table 2). Furthermore, quantitative real-time PCR showed 40-and 400-fold fungal quantity in bark and needles, respectively in comparison to wood.
Our results, therefore, suggest that interactions occur between different fungal species inhabiting Taxus that result in increased fungal Taxol biosynthesis. Prior results showed the presence of several fungi that inhabit Taxus plants (Farr and Rossman, 2006) but these were not tested for their effects on endophytic Taxol production. However, Taxol-producing endophytes have previously been shown to inhibit growth of non-WDF plant pathogens, including Pythium, Aphanomyces, and Phytophthora (Wang et al., 2000; Strobel, 2002, 2003; Strobel and Daisy, 2003). Our result is also consistent with previous studies showing that fungal metabolism can be affected by the metabolites of other fungi (Gayed, 1963). In the future, fractionation based bioassays using the fungi identified here as stimulating fungal Taxol production, may be used to identify candidate diffusible metabolites that affect fungal Taxol biosynthesis. It will then be useful to determine whether these compounds are also present in Taxus bark alcoholic extracts as hypothesized in this manuscript.

A greater number of previous studies have focused on fungalplant interactions than fungal-fungal interactions within plants. For example, several studies showed that fungi or fungal metabolites such as chitin can elicit plant Taxol, though the mechanism was not investigated (Wang et al., 2001; Li and Tao, 2009; Zhang et al., 2011). Endophytic fungi have also been shown to stimulate rate-limiting enzymes in the terpenoid pathway, namely 3-hydroxy-3-methylglutaryl coenzyme A reductase (HMGR) and 1-deoxy-D-xylulose 5-phosphate synthase (DXR; Gao et al., 2011). Arbuscular mycorrhizal (AM) fungi have also been shown to stimulate biosynthesis of plant terpenoids, alkaloids, and phenolics (Zhao and Yan, 2006). In this context, in the future, it will also be interesting to explore the possibility that compounds derived from endophytic fungi or other resident fungi may be directly interacting with the plant-derived Taxol pathway.

In conclusion, this study suggests that fungal endophytes may interact with other resident fungi to stimulate secondary metabolite production. The extent of this interaction in planta needs to be analyzed.
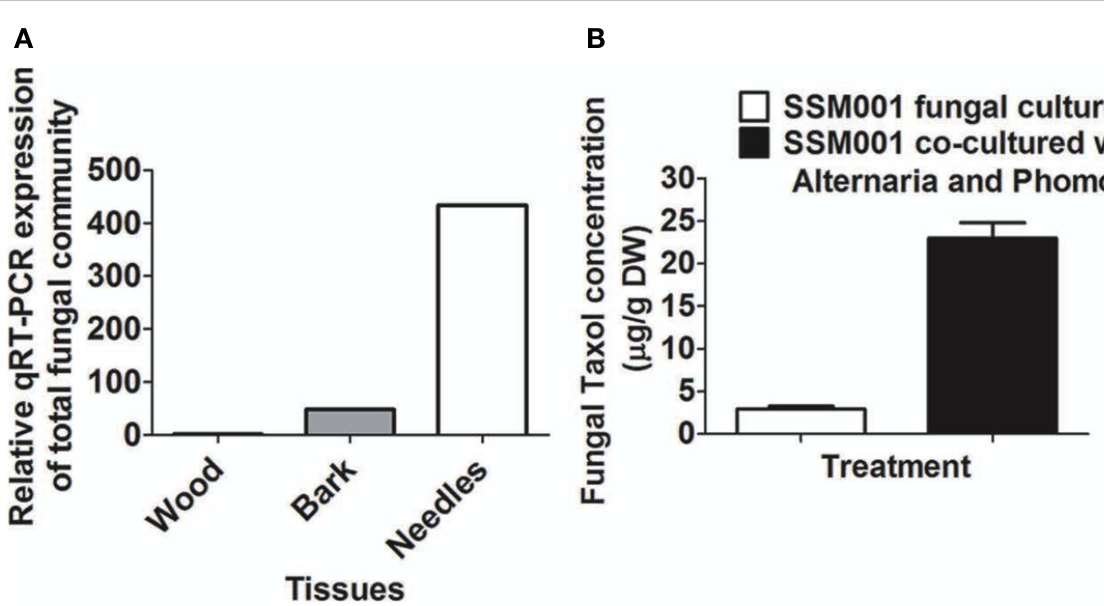

FIGURE 4 | Expression ratio of total fungal community in Taxus needles in comparison to Taxus bark and wood. (A) Expression ratio of total fungal community in Taxus needles and bark in comparison to Taxus

wood. (B) Effect of co-culturing of Alternaria and Phomopsis fungi isolated from outer bark and needles, respectively on SSM001 Taxol production in liquid culture. 


\section{ACKNOWLEDGMENTS}

We thank Dr. Rong Cao (Agriculture and Agri-Food Canada) for assistance with HPLC analysis, Lewis Melville and Dr. Larry Peterson (University of Guelph, ON, Canada) for assistance with microscopy, and Angela Holliss (Genome Facility, University of
Guelph) for help with tRFLP analysis. This research was supported by a generous scholarship from the Government of Egypt to Sameh S. M. Soliman and from NSERC Discovery and Canadian Foundation for Innovation grants to Manish N. Raizada.

\section{REFERENCES}

Akiyama, K., and Hayashi, H. (2006). Strigolactones: chemical signals for fungal symbionts and parasitic weeds in plant roots. Ann. Bot. 97, 925-931.

Amborabé, B.-E., Fleurat-Lessard, P., Chollet, J.-F., and Roblin, G. (2002). Antifungal effects of salicylic acid and other benzoic acid derivatives towards Eutypa lata: structureactivity relationship. Plant Physiol. Biochem. 40, 1051-1060.

Arthur, G. F.-N., Stewart, J. M., Sherwin, A. N., Jason, J. P., and Frank, D. (1994). Improved taxol yield by aromatic carboxylic acid and amino acid feeding to cell cultures of Taxus cuspidata. Biotechnol. Bioeng. 44, 967-971.

Booker, J., Sieberer, T., Wright, W., Williamson, L., Willett, B., Stirnberg, P., et al. (2005). MAX1 encodes a cytochrome $\mathrm{P} 450$ family member that acts downstream of MAX3/4 to produce a carotenoid-derived branch-inhibiting hormone. Dev. Cell 8, 443-449.

Brewer, P. B., Dun, E. A., Ferguson, B. J., Rameau, C., and Beveridge, C. A. (2009). Strigolactone acts downstream of auxin to regulate bud outgrowth in Pea and Arabidopsis. Plant Physiol. 150, 482-493.

Deckert, J., Melville, H., and Peterson, L. (2001). Structural features of a lophodermium endophyte during the cryptic life-cycle phase in the foliage of Pinus strobes. Mycol. Res. 105, 991-997.

Evensen, P. C., Solheim, H., Høiland, K., and Stenersen, J. (2000). Induced resistance of Norway spruce, variation of phenolic compounds and their effects on fungal pathogens. For. Pathol. 30, 97-108.

Fang, W., and Bidochka, M. J. (2006). Expression of genes involved in germination, conidiogenesis and pathogenesis in Metarhizium anisopliae using quantitative real-time Rt-PCR. Mycol. Res. 110, 1165-1171.

Farr, D. F., and Rossman, A. Y. (2006). Fungal Databases [Online]. Available at: http://nt.arsgrin.gov/fungaldatabases/ [accessed October 23, 2012].

Gamborg, O. L., Miller, R. A., and Ojima, K. (1968). Nutrient requirements of suspension cultures of soybean root cells. Exp Cell Res. 50, 151-158.

Gao, F.-K., Yong, Y.-H., and Dai, C.C. (2011). Effects of endophytic fungal elicitor on two kinds of terpenoids production and physiological indexes in Euphorbia pekinensis suspension cells. J. Med. Plants Res. 5, 4418-4425.

Gayed, S. K. (1963). On the interaction in culture between two pathogenic soil-inhabiting fungi. Mycopathologia 19, 205-215.

Gibson, D., Ketchum, R., Vance, N., and Christen, A. (1993). Initiation and growth of cell lines of Taxus brevifolia (Pacific yew). Plant Cell Rep. 12, 479-482.

Guo, B. H., Wang, Y. C., Zhou, X. W., Hu, K., Tan, F., Miao, Z. Q., et al. (2006). An endophytic taxol-producing fungus BT2 isolated from Taxus chinensis var. mairei. Afr. J. Biotechnol. 5, 875-877.

Han, J., Li, Z., Liu, B., Wang, H., Li, G., and Ye, H. (2007). Metabolic engineering of terpenoids in plants. Sheng Wu Gong Cheng Xue Bao 23, 561-569.

Haugland, R. A., Brinkman, N., and Vesper, S. J. (2002). Evaluation of rapid DNA extraction methods for the quantitative detection of fungi using real-time PCR analysis. J. Microbiol. Methods 50, 319-323.

Jacek, M., Magdalena, K., Kamil, G., Jacek, H., and Andrzej, P. (2007). A new catalytic activity from tobacco converting 2-coumaric acid to salicylic aldehyde. Physiol. Plantarum 129, 461-471.

Kang, S.-M., Jung, H.-Y., Kang, Y.-M., Yun, D.-J., Bahk, J.-D., Yang, J.-K., et al. (2004). Effects of methyl jasmonate and salicylic acid on the production of tropane alkaloids and the expression of PMT and $\mathrm{H} 6 \mathrm{H}$ in adventitious root cultures of Scopolia parviflora. Plant Sci. 166, 745-751.

Li, J.-Y., Sidhu, R. S., Bollon, A. R. T., and Strobel, G. A. (1998a). Stimulation of taxol production in liquid cultures of Pestalotiopsis microspora. Mycol. Res. 102, 461-464.

Li, J. Y., Sidhu, R. S., Ford, E. J., Long, D. M., Hess, W. M., and Strobel, G. A. (1998b). The induction of taxol production in the endophytic fungus-Periconia sp from Torreya grandifolia. J. Ind. Microbiol. Biotechnol. 20, 259-264.

Li, J. Y., Strobel, G., Sidhu, R., Hess, W. M., and Ford, E. J. (1996). Endophytic taxol-producing fungi from bald cypress, Taxodium distichum. Microbiology 142, 2223-2226.

Li, Y.-C., and Tao, W.-Y. (2009). Interactions of taxol-producing endophytic fungus with its host (Taxus spp.) during Taxol accumulation. Cell Biol. Int. 33, 106-112.

Li, Y.-C., Tao, W.-Y., and Cheng, L. (2009). Paclitaxel production using co-culture of Taxus suspension cells and paclitaxel-producing endophytic fungi in a co-bioreactor. Appl. Microbiol. Biotechnol. 83, 233-239.

Mattsson, J., Sung, Z. R., and Berleth, T. (1999). Responses of plant vascular systems to auxin transport inhibition. Development 126, 2979-2991.

Metz, A. M., Haddad, A., Worapong, J., Long, D. M., Ford, E. J., Hess, W. M. et al. (2000). Induction of the sexual stage of Pestalotiopsis microspora, a taxol-producing fungus. Microbiology 146, 2079-2089.

Moon-Jong, N., Jae-Gwon, Y., KyungSoo, K., Young-Mean, Y., KyoungAe, K., Hee-Yong, H., et al. (1999). Isolation of a novel microorganism, Pestalotia heterocornis, producing paclitaxel. Biotechnol. Bioeng. 64 620-623.

Nakamura, T., Mitsuoka, K., Sugano, M., Tomita, K., and Murayama, T. (1985). Effects of auxin and gibberellin on conidial germination and elongation of young hyphae in Gibberella fujikuroi and Penicillium notatum. Plant Cell Physiol. 26 1433-1437.

Nims, E., Dubois, C. P., Roberts, S. C. and Walker, E. L. (2006). Expression profiling of genes involved in paclitaxel biosynthesis for targeted metabolic engineering. Metab. Eng. 8, 385-394.

Noh, M. J., Yang, J. G., Kim, K. S., Yoon, Y. M., Kang, K. A., Han, H. Y., et al. (1999). Isolation of a novel microorganism, Pestalotia heterocornis, producing paclitaxel. Biotechnol. Bioeng. 64, 620-623.

Pastírová, A., Repcák, M., and Eliasová, A. (2004). Salicylic acid induces changes of coumarin metabolites in Matricaria chamomilla L. Plant Sci. 167, 819-824.

Perez-Vera, O., Yanez-Morales, M., Alvarado-Rosales, D., Cibrian-Tovar, D., and Garcia-Diaz, S. (2005). Fungi associated to eucalyptus, Eucalyptus grandis Hill ex Maid. Agrociencia 39, 311-318.

Roberts, S. C. (2007). Production and engineering of terpenoids in plant cell culture. Nat. Chem. Biol. 3, 387-395.

Seppänen, S. K., Syrjälä, L., Weissenberg, K., Teeri, T. H., Paajanen, L., and Pappinen, A. (2004). Antifungal activity of stilbenes in in vitro bioassays and in transgenic Populus expressing a gene encoding pinosylvin synthase. Plant Cell Rep. 22, 584-593.

Soliman, S. S. M., Tsao, R., and Raizada, M. N. (2011). Chemical inhibitors suggest endophytic fungal paclitaxel is derived from both mevalonate and non-mevalonate-like pathways. J. Nat. Prod. 74, 2497-2504.

Staniek, A., Woerdenbag, H. J., and Kayser, O. (2009). Taxomyces andreanae: a presumed paclitaxel producer demystified? Planta Med. 75, 1561-1566.

Stierle, A., Strobel, G., and Stierle, D. (1993). Taxol and taxane production by Taxomyces andreanae, an endophytic fungus of Pacific yew. Science 260, 214-216.

Stierle, A., Strobel, G., Stierle, D., Grothaus, P., and Bignami, G. (1995). The search for a taxolproducing microorganism among the endophytic fungi of the Pacific yew, Taxus brevifolia. J. Nat. Prod. 58, 1315-1324.

Strobel, G., and Daisy, B. (2003). Bioprospecting for microbial endophytes and their natural products. Microbiol. Mol. Biol. Rev. 67, 491-502.

Strobel, G., Yang, X., Sears, J., Kramer, R., Sidhu, R. S., and Hess, W. M. (1996a). Taxol from Pestalotiopsis microspora, an endophytic fungus of Taxus wallachiana. Microbiology 142, 435-440.

Strobel, G. A., Hess, W. M., Ford, E., Sidhu, R. S., and Yang, X. (1996b). Taxol from fungal endophytes and the issue of biodiversity. J. Ind. Microbiol. Biotechnol. 17, 417-423. 
Strobel, G. A. (2002). Rainforest endophytes and bioactive products. Crit. Rev. Biotechnol. 22, 315-333.

Strobel, G. A. (2003). Endophytes as sources of bioactive products. Microbes Infect. 5, 535-544.

Swanty, G. (2000). Defense signaling in plants. Resonance 5, 43-53.

Välimaa, A.-L., HonkalampiHämäläinen, U., Pietarinen, S., Willför, S., Holmbom, B., and Von Wright, A. (2007). Antimicrobial and cytotoxic knotwood extracts and related pure compounds and their effects on food-associated microorganisms. Int. J. Food Microbiol. 115, 235-243.

Veeresham, C., Mamatha, R., Babu, C. P., Srisilam, K., and Kokate, C. K. (2003). Production of Taxol and its analogues from cell cultures of Taxus wallichiana. Pharm. Biol. 41, 426-430.

Wang, C., Wu, J., and Mei, X. (2001). Enhancement of taxol production and excretion in Taxus chinensis cell culture by fungal elicitation and medium renewal. Appl. Microbiol. Biotechnol. 55, 404-410.

Wang, J., Li, G., Lu, H., Zheng, Z., Huang, Y., and Su, W. (2000). Taxol from Tubercularia sp. strain TF5, an endophytic fungus of Taxus mairei.
FEMS Microbiol. Lett. 193, 249-253.

Wang, Y.-D., Wu, J.-C., and Yuan, Y.-J. (2007). Salicylic acid-induced taxol production and isopentenyl pyrophosphate biosynthesis in suspension cultures of Taxus chinensis var. mairei. Cell Biol. Int. 31, 1179-1183.

Wang, Y.-D., Yuan, Y.-J., and Wu, J.-C. (2004). Induction studies of methyl jasmonate and salicylic acid on taxane production in suspension cultures of Taxus chinensis var. mairei. Biochem. Eng. J. 19, 259-265.

Wu, H.-S., Raza, W., Fan, J.-Q., Sun, Y.-G., Bao, W., Liu, D.-Y., et al. (2008). Antibiotic effect of exogenously applied salicylic acid on in vitro soilborne pathogen, Fusarium oxysporum f. sp. niveum. Chemosphere 74, 45-50.

Xu, F., Tao, W., Cheng, L., and Guo, L. (2006). Strain improvement and optimization of the media of taxolproducing fungus Fusarium maire. Biochem. Eng. J. 31, 67-73.

Yoneyama, K., Xie, X., Kusumoto, D., Sekimoto, H., Sugimoto, Y., Takeuchi, Y., et al. (2007). Nitrogen deficiency as well as phosphorus deficiency in sorghum promotes the production and exudation of 5-deoxystrigol, the host recognition signal for arbuscular mycorrhizal fungi and root parasites. Planta 277, 125-132.

Young, D. H., Michelotti, E. L., Swindell, C. S., and Krauss, N. E. (1992). Antifungal properties of taxol and various analogues. Experientia 48, 882-885.

Yu, T., Zhang, H., Li, X., and Zheng, X. (2008). Biocontrol of Botrytis cinerea in apple fruit by Cryptococcus laurentii and indole-3-acetic acid. Biol. Control 46, 171-177.

Zhang, J.-F., Gong, S., and Guo, Z.G. (2011). Effects of different elicitors on 10-deacetylbaccatin III10-O-acetyltransferase activity and cytochrome P450 monooxygenase content in suspension cultures of Taxus cuspidata cells. Cell Biol. Int. Rep. 18, 7-13.

Zhao, K., Ping, W., Zhang, L., Liu, J. Lin, Y., Jin, T., et al. (2008). Screening and breeding of high taxol producing fungi by genome shuffling. Sci. China C Life Sci. 51, 222-231.

Zhao, X., and Yan, X. (2006). Effects of arbuscular mycorrhizal fungi on plant secondary metabolism. Acta Phytoecol. Sinica 30, 514-521.

Zhou, X., Zhu, H., Liu, L., Lin, J., and Tang, K. (2010). A review: recent advances and future prospects of taxol-producing endophytic fungi. Appl. Microbiol. Biotechnol. 86, 1707-1717.

Conflict of Interest Statement: The authors declare that the research was conducted in the absence of any commercial or financial relationships that could be construed as a potential conflict of interest.

Received: 19 November 2012; paper pending published: 15 December 2012; accepted: 02 January 2013; published online: 22 January 2013.

Citation: Soliman SSM and Raizada MN (2013) Interactions between cohabitating fungi elicit synthesis of Taxol from an endophytic fungus in host Taxus plants. Front. Microbio. 4:3. doi: 10.3389/fmicb.2013.00003

This article was submitted to Frontiers in Fungi and Their Interactions, a specialty of Frontiers in Microbiology.

Copyright () 2013 Soliman and Raizada. This is an open-access article distributed under the terms of the Creative Commons Attribution License, which permits use, distribution and reproduction in other forums, provided the original authors and source are credited and subject to any copyright notices concerning any third-party graphics etc. 